\title{
High frequency magnetization dynamics metrology using a pulsed field inductive microwave magnetometer
}

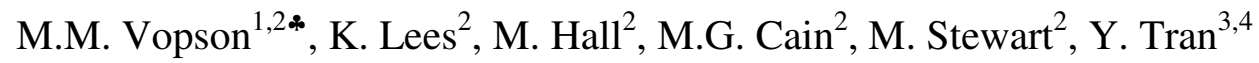 \\ ${ }^{1}$ University of Portsmouth, Faculty of Science, SEES, Burnaby Building, Portsmouth PO1 3QL, UK \\ ${ }^{2}$ National Physical Laboratory, Teddington TW11 0LW, UK \\ ${ }^{3}$ University of Exeter, Harrison Building, Exeter, EX4 4QF, UK \\ ${ }^{4}$ Plasma Quest Ltd, Hook, RG27 1QT, UK
}

We report the development of a pulsed inductive microwave magnetometer for measurements of ultra fast magnetization dynamics of soft magnetic thin films. This article details the instrument construction and the measurement procedure. We show experimentally that our instrument detects nano-second and sub-nano second magnetization precession / relaxation processes, in real time, without the need of data averaging, interpolation or any other post measurement data processing. This enhanced detection resolution has been achieved by careful design and optimization of the coplanar waveguide, which will be described in detail in this article. Moreover, we show that, unlike other similar instruments, our apparatus is significantly simplified, reducing the cost as well as the measurement procedure.

\section{Introduction}

Switching the magnetization direction of a macroscopic ferromagnetic structure looks, on the timescale of microseconds or larger, as an uniform and instantaneous process. However, at the speed of reading / writing digital information in modern magnetic data storage devices, the data access takes place in nanoseconds, where the dynamic of the magnetization vector is more complicated.

The lowest energy state requires that the equilibrium direction of the magnetic spin is parallel with the applied magnetic field lines. This also applies to a macro-spin, magnetic moment or simply the total magnetization of a sample. If a sudden change of the orientation of the applied magnetic field occurs, or similarly if an additional magnetic field is applied in a different direction, a new equilibrium direction will result. Consequently, magnetization (M) will try to realign to this new direction. Due to the misalignment between the two vectors, a torque will act on $\mathrm{M}$ perpendicular to the plane formed by the magnetic field and $\mathrm{M}$ itself. The dynamics of $\mathrm{M}$ under the action of the torque will be a damped precessional motion, as resulted from the Landau-Lifshitz-Gilbert (LLG) equation [1,2]. The phenomenological damping parameter introduced by Gilbert accounts for the energy dissipation channels resulting from the fact that $\mathrm{M}$ is not isolated and it is coupled to the environment (other spins, lattice, charge carriers, etc). In general, the damping parameter dictates the total relaxation time during the switching process. However, in the case of ultra-short applied field pulses, the relaxation time is dominated by the details of the magnetic precession $[3,4]$. The precise understanding of the magnetization dynamics at ultrafast frequencies is critical for the functionality of magnetic data storage devices as well as for fundamental advances in the solid-state physics of systems at microwave frequencies. Suitable metrologies are therefore required for such measurements of existing magnetic materials as well as new emerging materials such as multiferroics and spintronic structures. A number of experimental techniques dedicated to the study

\footnotetext{
* Formerly known as M. Vopsaroiu, Correspondence: melvin.vopson@port.ac.uk
} 
of fast magnetization dynamics have been successfully developed. They all involve some kind of pulsed excitation with a short (fs to ns) magnetic field pulse followed by the interrogation of the sample's response in the time domain. The excitation mechanisms, as well as the measurement of the sample's response are determined either electrically using a coplanar waveguide (CPW) system [5,6] or excite electrically and probe optically using fs pumped-probe MOKE measurements [7,8,]. Other techniques of studying fast magnetization dynamics based on time dependent X-ray resonant magnetic scattering [9], ultra short 2-5 ps magnetic field pulses as high as $20 \mathrm{~T}$ achieved at linear e-beam accelerators [10], or magneto-transport response to ultra-short magnetic pulses [11,12] have also been reported. In this paper we present the experimental development of a pulse inductive microwave magnetometer (PIMM), which is an improved design of the original instrument introduced by Kos et al. [5]. Our improved design includes a few experimental simplifications, optimization of the coplanar waveguide for this particular experiment and the ability to perform accurate measurements without the requirement of post measurement data processing. The instrument has been experimentally tested using standard permalloy thin film samples.

\section{Experimental design and instrumentation}

\subsection{Pulsed inductive microwave magnetometer}

The dynamic of magnetization switching is very complex and magnetization reversal under applied magnetic fields takes place via a damped ultra-fast magnetization precession around the new equilibrium direction. During this precession, an inductive signal can be induced in a conductor located in the immediate proximity of the sample, via Faraday induction. Under the optimum experimental conditions, this signal can be experimentally measured, facilitating the study of ultra-fast magnetization dynamics. In this work we have developed such instrument, called "pulse inductive microwave magnetometer" (PIMM), which is based on Kos et al. [5] original design, with additional improvements and simplifications. Figure 1 shows a schematic diagram of the experimental setup, the coplanar waveguide (CPW) and the geometry of the applied fields. The CPW is the key component to this experiment as it facilitates not only the application of ultra-fast magnetic field pulses, but also it measures the inductive response from the sample. Both CPW and the sample are placed in the YZ plane, with the applied bias magnetic field in the OZ direction and the pulse field in the OY direction. In the case of thin magnetic films, the out-of-plane $\mathrm{M}_{\mathrm{x}}$ component of the magnetization is usually much smaller than the in-plane components. Therefore, $\mathrm{M}_{\mathrm{x}}$ contribution to the inductive signal is negligible with the only significant contribution given by the transverse $\mathrm{M}_{\mathrm{y}}$ component of the magnetization [6]. The measurement system has been tested experimentally on a 100 $\mathrm{nm} \mathrm{NiFe}$ thin film sample deposited on Si substrate using a HiTUS sputtering system [13]. The surface of the film was coated with $100 \mathrm{~nm}$ thin layer of photoresist using a spin coater. This is required to both protect the film's surface and to prevent it from electrically shorting the CPW conductors. The sample is then placed on the CPW with the magnetic film facing the CPW plane. The characteristic impedance of the CPW is $50(+/-0.5) \mathrm{Ohm}$ and the bandwidth is greater than $10 \mathrm{GHz}$. 
This CPW design allows us to measure thin film square samples of up to $20 \mathrm{~mm}$ size. Due to its critical importance for the functionality of the instrument, the practical aspects of the CPW will be discussed in detail in the next section. The Picosecond Pulse Generator (Picosecond Model 10,050A) provides 50 ps rise-time pulses to the CPW, which in turn generates fast pulses of magnetic fields that couple to the magnetic thin film sample located in the close proximity of the CPW. The pulse generator also provides $10 \mathrm{~V}$ amplitude pulses with adjustable width from 100 ps to $10 \mathrm{~ns}$, pulse repetition rate $100 \mathrm{~Hz}$ to 100 $\mathrm{kHz}$ and output jitter of less than 1 ps rms. All our measurements have been performed at $10 \mathrm{kHz}$ pulse repetition rate. The $10 \mathrm{~V}$ amplitude pulse is attenuated via a wide bandwidth attenuator before it passes through the $\mathrm{CPW}$. The response is measured via a $20 \mathrm{GHz}$ Agilent (Infiniium DCA-J 86100C) digital sampling oscilloscope with 18 bit amplitude resolution and a noise level of $0.1 \mathrm{mV} \mathrm{rms}$. This is capable to capture the inductive signals induced in the CPW by the magnetization precession, when a perturbation from its equilibrium position is applied (i.e. the pulsed field). The time interval accuracy of the oscilloscope is better than $10 \mathrm{ps}$ and the combined jitter is less than 2.5 ps. All our acquired waveforms contain 4000 data points per waveform with 5 trace averages. The LabView software allows selecting a wide range of parameters, including the number of trace averages. Although our measurements are reported for 5 trace averages, we have not seen any significant difference between a single trace and 5 traces. A set of Helmholtz electromagnet coils has the function to provide the DC longitudinal bias magnetic field. The electromagnet is powered by a $1 \mathrm{~kW}$ bi-polar power supply and is capable of achieving magnetic fields of up to $23 \mathrm{kA} / \mathrm{m}(\sim 300 \mathrm{Oe})$. This is large enough to saturate most of known thin film magnetic materials, including the examined $\mathrm{NiFe}$ sample. A Hall probe instrument provides the exact dc bias field at the sample location. The CPW sample holder with the sample inside is placed carefully between the Helmholtz coils and the magnetic dc bias field is switched $\mathrm{ON}$ to a saturating level (>300 Oe). A reference waveform is acquired by applying a pulse field while the dc magnetic bias field is switched $\mathrm{ON}$ at the maximum level. The applied magnetic field is then reduced to a lower value suitable for measurements (typically $10-50 \mathrm{Oe}$ ). A 
second pulse field identical to the reference one is applied again to the sample and the second waveform is acquired. When examining the two acquired waveforms together, they look in fact identical. However, the second waveform contains the extremely small inductive signal induced by the magnetization precession during its motion to equilibrium. In order to extract the inductive magnetization relaxation signal, a simple subtraction of the two waveforms is performed directly during the measurement using a LabView code. The resulting signal shows the relaxation of magnetization in the time domain, which can be analysed to extract information regarding the damping constant and the relaxation time. Unlike the original design of Kos et al. [5], we have eliminated the need of the transverse Helmholtz coils required for the acquisition of the reference waveform. Instead, we established experimentally that a large enough magnetic field applied longitudinally is sufficient to acquire an excellent reference waveform. In our experiment, this field is supplied by the same electromagnet used to apply the low magnetic bias field during the measurement, except that the reference waveform is acquired in a very large, saturating applied field. This simplification reduces the size, the cost and the complexity of the instrument. Moreover, unlike most similar instruments, our measurement yields the magnetization relaxation data directly, without the need for additional complicated and time-consuming data processing, averaging or interpolations. We believe that this is because the CPW has been fully optimized to provide the best magnetic field profile and magnitude to the sample during the field pulses. This CPW optimization is discussed in detail in the next section.

\subsection{Coplanar waveguide (CPW) design and optimisation}

As already mentioned in the previous section, in order to optimize the instrument's functionality the CPW had to be designed and fabricated to specific standards. In particular, the optimization of the magnetic pulse field generated at the sample by the CPW has been the main objective of this study. Besides providing the excitation pulses and detection of the inductive relaxation response, the CPW also acts as the sample holder. In order to optimise its design, we used two different commercial software packages:

i) TXLINE for the geometric and material optimisation of the CPW, imposing 50 Ohm impedance at $10 \mathrm{GHz}$ operation frequency;

ii) Microwave Studio for the calculation of the magnetic field profile and amplitude of the CPW in operational conditions;

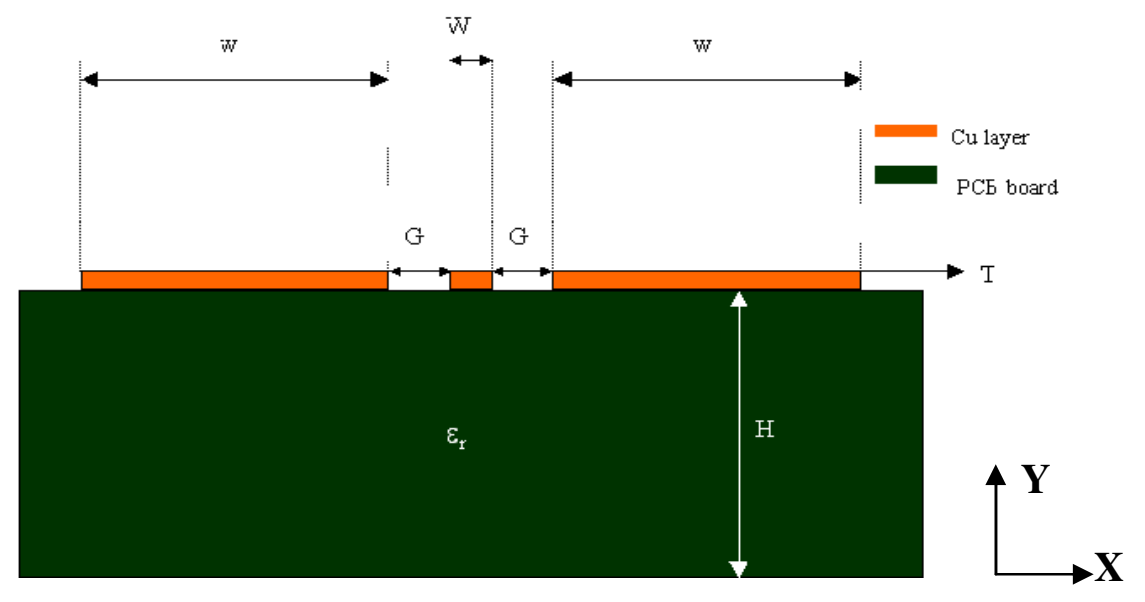

Figure 2. Schematic diagram of the CPW in cross section. 
The calculations correspond to the following set of parameters and materials: alumina substrate with $\varepsilon_{\mathrm{r}}=9.8$, operation frequency 10 $\mathrm{GHz}$, substrate thickness $\mathrm{H}$ $=3 \mathrm{~mm}$, CPW length 35 $\mathrm{mm}$, CPW width $20 \mathrm{~mm}$, metallic electrodes made of $\mathrm{Au}$, the width of the ground electrodes $\mathrm{w}=10$ $\mathrm{mm}$. We have performed the modelling by imposing the $50 \mathrm{Ohm}$ impedance of the CPW for a set of 4 different thicknesses of the

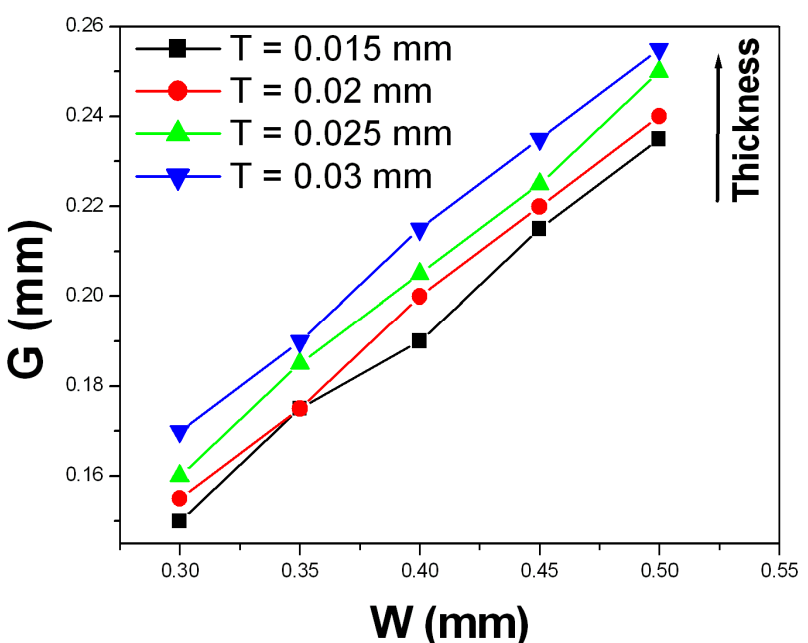

Figure 3. Gap size versus the central stripe width for different thicknesses when the CPW impedance is kept constant at $50 \mathrm{Ohm}$ metallic electrodes: $\mathrm{T}=15,20,25$ and $30 \mu \mathrm{m}$, respectively. Figure 2 shows a cross section of the CPW including the main modelling parameters. Using TXLINE and imposing constant impedance of $50 \mathrm{Ohm}$, we have addressed the following question:

What is the optimum gap size for a set of central stripe widths and different thickness of the metallic electrodes?

Figure 3 shows the gap size calculated for specific stripe width ( $\mathrm{W}=300,350,400$, 450 and $500 \mu \mathrm{m}$, respectively) and four different thicknesses of the metallic electrodes.

Having optimized the CPW dimensions, these values were then plugged in the Microwave Studio modelling package in order to optimise the magnetic field generated by the CPW. Figure 4.B shows the calculated field values at a fixed distance of $300 \mu \mathrm{m}$ above the CPW at its centre position. The calculations are performed for each thickness and each central line width. The distance of $300 \mu \mathrm{m}$ has been chosen as this corresponds most likely to the sample - CPW separation distance during the measurements. According to the modelling data, the largest magnetic fields appear to be obtained for the thicker metallic electrodes, i.e. $25-30 \mu \mathrm{m}$. Although not fully understood, this important result was fully adopted in our CPW design. The stripe line width also appears to have an effect on the optimal magnetic field generated. Although the difference in the magnitude of the field is very small, the field peaks at around 350 - $400 \mu \mathrm{m}$ stripe line width, which again has been adopted in our CPW design parameters.

Calculated maximum fields at the air / metallic stripe line interface can be as high as $100 \mathrm{~A} / \mathrm{m}(1.25 \mathrm{Oe})$ at the centre of the CPW and substantially larger at the edges. Figure 4.A shows a typical example of the calculated field profile for both $\mathrm{x}$ and $\mathrm{y}$ components of the magnetic field, obtained using Microwave Studio modelling package. Based on the modelling results, the following CPW design parameters have been established: Metallic Au electrodes with thickness, $\mathrm{T}=30 \mu \mathrm{m}$; Central stripe 
line width $=400 \mu \mathrm{m}$; Gap size $=210 \mu \mathrm{m}$; Substrate alumina or YAG dielectric $\left(\varepsilon_{\mathrm{r}} \approx\right.$ 10); Substrate thickness $=3 \mathrm{~mm}$; Total length of the CPW $=35 \mathrm{~mm}$; Total width of the $\mathrm{CPW}=20 \mathrm{~mm}$.

A)

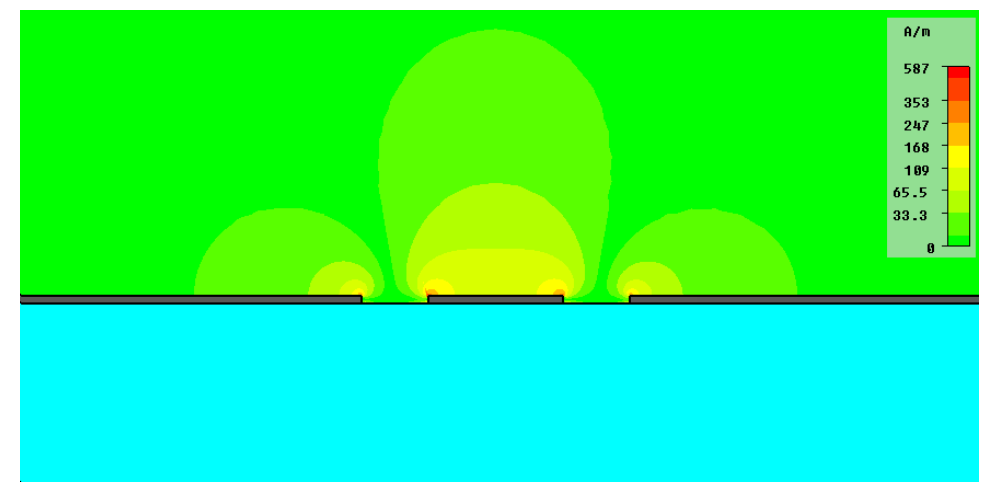

B)

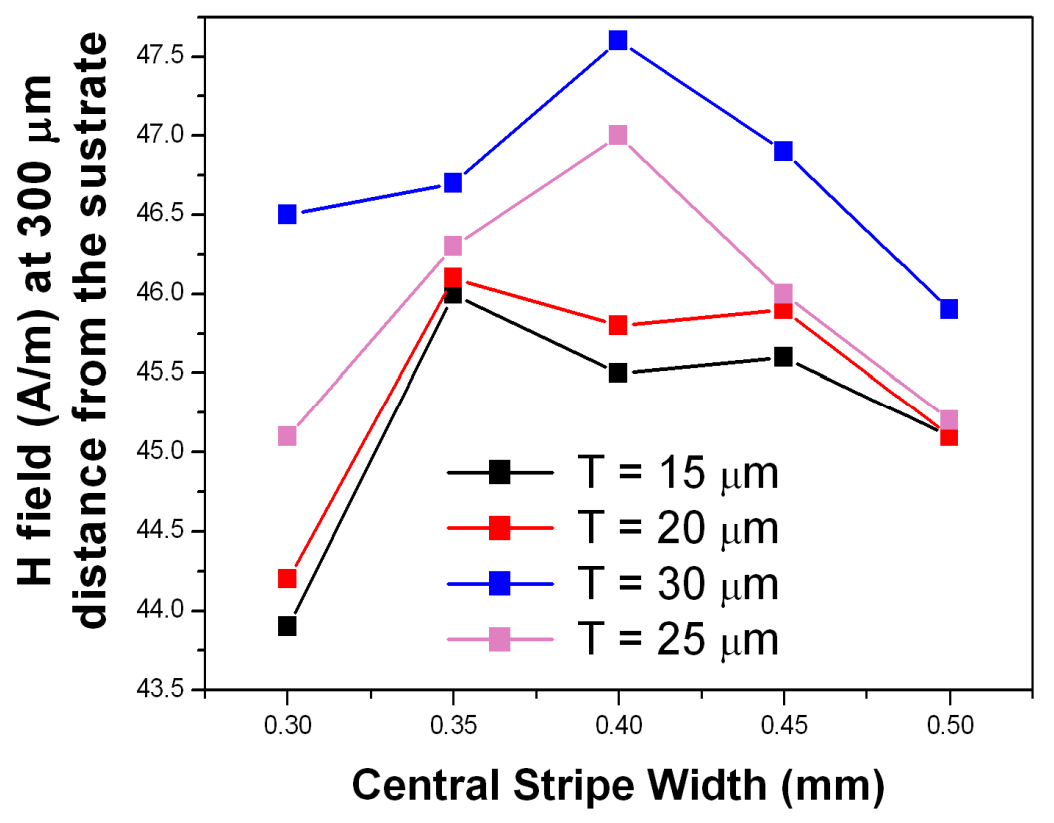

Figure 4. A) Cross section of the CPW with the magnetic field profiles calculated using Microwave Studio; B) Calculated magnetic field amplitude versus central stripe width for different thicknesses.

\section{Measurement procedure}

The instrument is operated via the following experimental procedure:

a) The sample is placed inside the cavity sample holder, with the magnetic film facing the CPW plane, as close as possible in order to achieve the best measurement sensitivity. The sample must be located at the centre of the $\mathrm{CPW}$ with the central stripe line along the central axis of the sample. This will ensure the most uniform and effective field profile to the sample.

b) The CPW sample holder with the sample inside is then placed carefully inside the Helmholtz coils, exactly at the middle point between the coils. 
c) The magnetic dc bias field is switched $\mathrm{ON}$ to a saturating level ( $>300 \mathrm{Oe}$ ) by applying maximum voltage / current to the Helmholtz coils from the bi-polar power supply.

d) A reference waveform is acquired by applying a pulse field while the dc magnetic field bias is switched $\mathrm{ON}$ at the maximum level.

e) The applied magnetic field is then reduced to a lower value suitable for measurements (typically $10-50 \mathrm{Oe}$ ).

f) A second pulse field identical to the reference one is applied again to the sample and the second waveform is acquired.

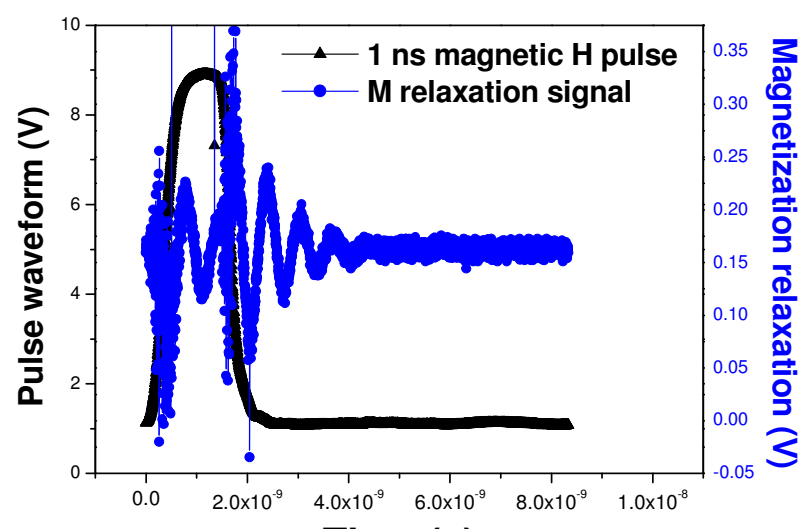

Time (s)

Figure 5. Experimental data showing the magnetic 1 ns pulse signal (black) and the inductive relaxation signal at the CPW given by the magnetization precession (no signal attenuator used this time).

In order to extract the inductive magnetization relaxation signal, a subtraction of the two waveforms is performed. The measurement is fully automated using LabView and the data is outputted directly without the need for additional data processing, averaging or interpolations, which is a substantial improvement in comparison to other similar instruments.

After the subtraction procedure is applied, the damped magnetization precession is clearly visible as a sequence of decaying oscillations at the end of a typical pulse. The resulting signal shows the magnetization relaxation in the time domain, which can be analysed to extract the damping constant, the relaxation time and other useful parameters (see figure 5).

\section{Testing possible artefacts}

In order to rule out possible artefacts, a simple test has been performed. First, a standard 75 $\mathrm{nm} \mathrm{NiFe}$ thin film sample has been tested using the previously defined measurement procedure. This measurement was followed by another measurement of a $\mathrm{Si}$ wafer test specimen cut to identical size to the NiFe sample and also coated with a PMMA layer of identical thickness to

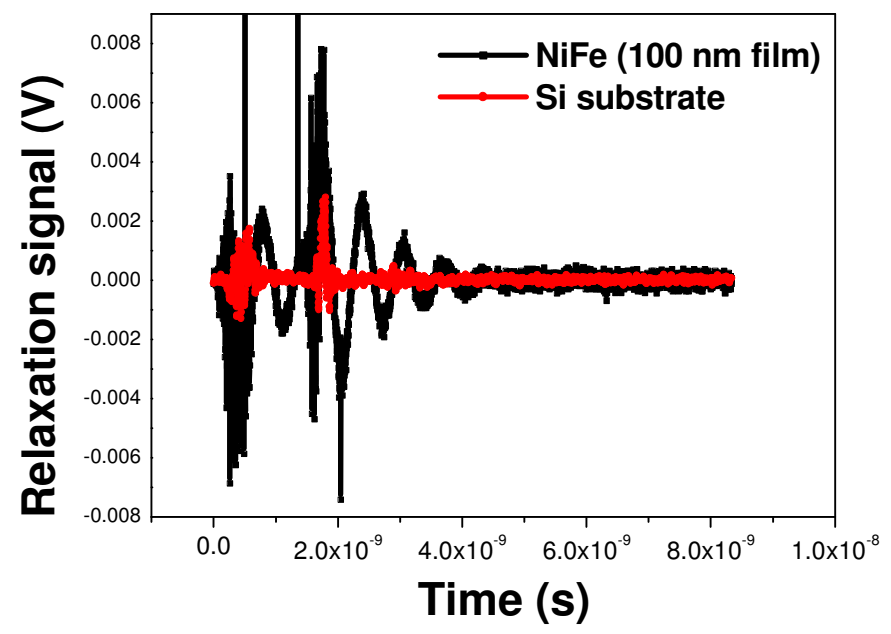

Figure 6. Testing measurement artefacts by comparing the response of a magnetic thin film with that of a $\mathrm{Si}$ substrate of similar shape tested under identical conditions.

that of the NiFe film. The Si test specimen has been inserted in the microwave cavity at the same location as the $\mathrm{NiFe}$ sample and tested under identical conditions. Figure 
6 shows the $1 \mathrm{~ns}$ waveform pulse and the magnetization relaxation data obtained after employing the subtraction procedure for both samples. The data shows clearly the oscillatory relaxation signal related to the magnetization precession of the NiFe thin film and a flat response from the non-magnetic test specimen sample.

Additional tests, not shown here, ruled out beyond any doubt the measurement artefacts. These included: a) no sample present; b) sample under various applied DC magnetic field bias conditions; c) sample under various baseline waveform measurement procedures. Small variations in the $50 \mathrm{Ohm}$ characteristic impedance of the CPW can occur due to the SMA connectors between the coaxial cable and the $\mathrm{CPW}$, as well as due to the sample itself, which modifies slightly the effective dielectric permittivity of CPW. Such small impedance mismatches will cause reflections, which in turn will produce an additional inductive response. However, for our measurements these additional signals are irrelevant as they are subtracted out via the reference waveform, as described in the measurement procedure, section 3.

\section{Repeatability test}

Repeatability is a particular issue that must be addressed when developing any type of instrumentation. This is very important because it helps to distinguish between time dependent system drifts and real physical effects, which are particularly important in ferromagnetic systems. The repeatability test of our instrument involved the sequential measurement when a particular sample was kept in the same position and measurements were taken at different time intervals under identical conditions. Figure 7 shows the data corresponding to a 10 ns pulse measured at time intervals ranging from 0 to 102 minutes. The data indicates full repeatability of the measurement, with perfect overlapping of the measured waveforms.

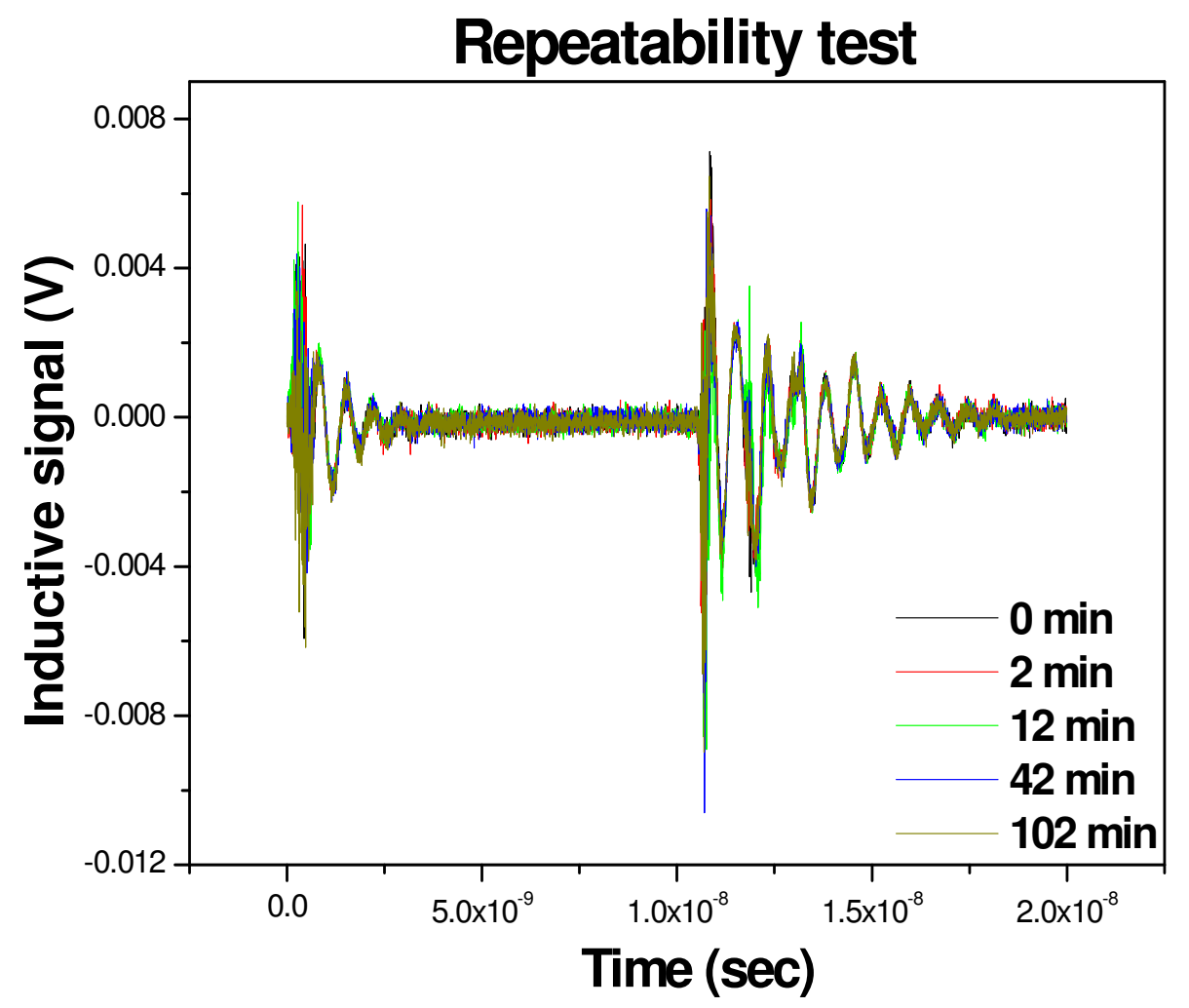

Figure 7. Repeatability test performed on the same sample $(100 \mathrm{~nm}$ $\mathrm{NiFe}$ film) at different time intervals under identical measurement 
However, another test has been taken with a time interval of 24 hours. Figure 8 shows the comparison between data acquired at $\mathrm{t}=0$ and 24 hours later, while all instruments were also switched off over night. On this occasion, the data indicates a time shift between the initial waveform and the one measured 24 hours later. The inserts in the figure 8 show in detail the regions at the beginning and the end of the pulse, where the magnetization relaxation occurs. A close examination of the data reveals a time shift of 113 ps for the main relaxation at the end of the pulse, and $56 \mathrm{ps}$ for the relaxation occurring at the beginning of the pulse. In both cases, the jitter noise is around \pm 15 ps. It is not clear what the cause of the time shift is, but this could be related to slight temperature variations in the laboratory during the $24 \mathrm{~h}$ interval, or due to a slight microscopic movement of the sample. Although significant, this time drift is only around $1.5 \%$ of the total relaxation time.

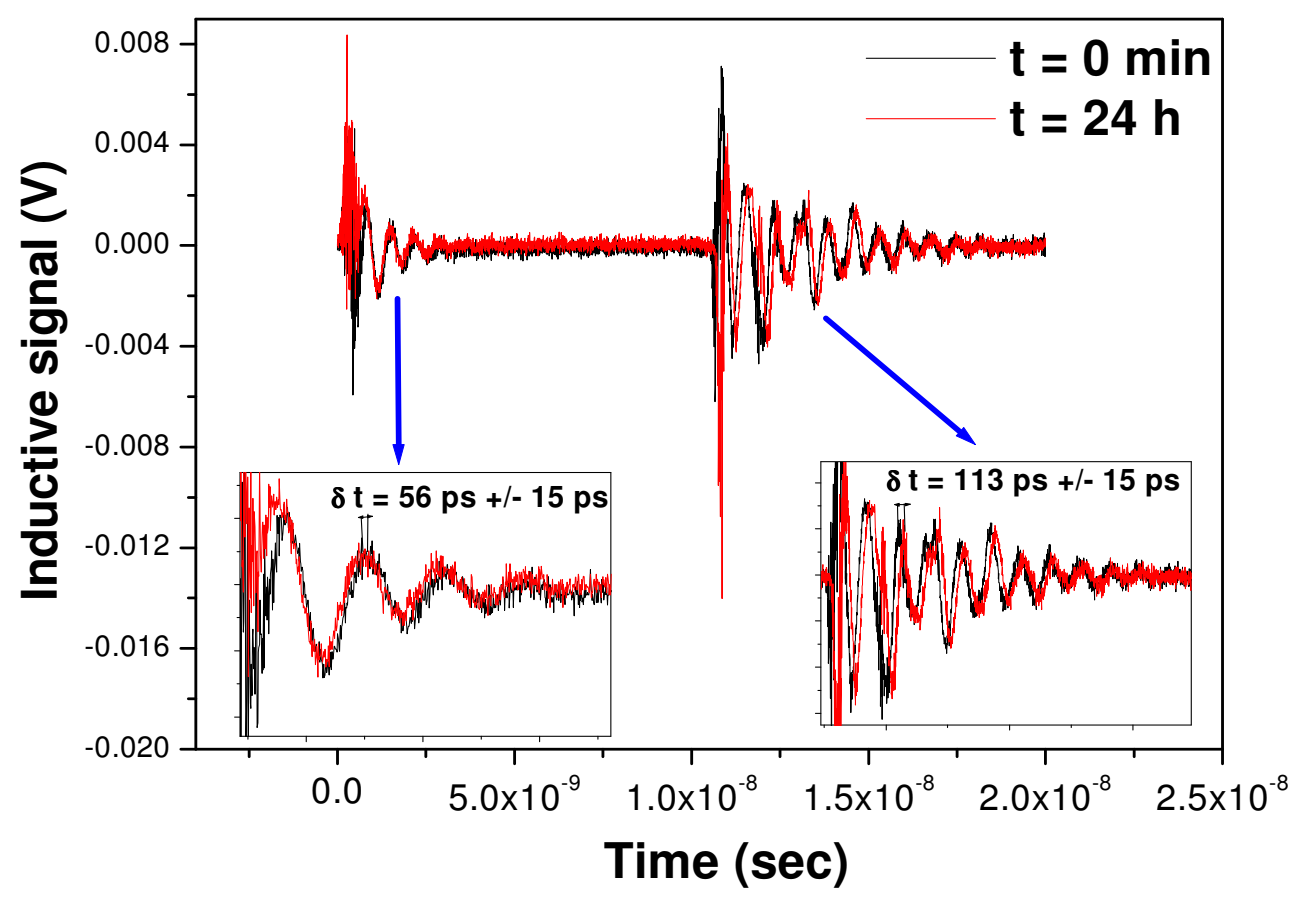

Figure 8. Repeatability test performed on the same sample under identical measurement conditions, after 24 hours switch OFF procedure.

\section{Data analysis}

Figures 5 and 6 show a typical magnetization relaxation response to $1 \mathrm{~ns}$ applied pulse field. For all our measurements, the sample has been biased by a 20 Oe longitudinally applied magnetic field. The data indicates a relaxation time of around $3 \mathrm{~ns}$ after the termination of the pulse. However, at a closer inspection, ringing appears to occur inside the pulse. This is more obvious in figures 7 and 8, where longer, $10 \mathrm{~ns}$ pulses, were used in the experiment. The data clearly shows a relaxation at the end of the applied pulse, and an additional precession at the beginning of it. This is more obvious when the applied pulse duration is longer than the relaxation time of magnetization. Examining the data in figure 7, the two precession signals appear to have different relaxation times. The first relaxation at the beginning of the pulse is about $\sim 3 \mathrm{~ns}$, while the second relaxation at the end of the $10 \mathrm{~ns}$ pulse is around $7 \mathrm{~ns}$. The amplitudes of the two relaxations also appear to be different, with the amplitude 
of the first precession smaller than that of the second one. This result can be explained in terms of two independent precession mechanisms. Figure 9 shows again the inductive response of a $\mathrm{NiFe} 100 \mathrm{~nm}$ film under a 20 Oe bias applied longitudinal field response to the $10 \mathrm{~ns}$ excitation pulse. For better clarity, this time the pulse is also plotted together with the inductive relaxation response. Initially, the magnetization vector is in equilibrium along the bias field direction. Upon the application of the pulse field transversal to the bias field, the $M$ vector rotates to the new equilibrium direction given by the vector resultant between the two acting fields (bias field and pulse field). As predicted by the LLG equation, this rotation to a new equilibrium direction takes place via a precession and corresponds to the first relaxation signal measured at the beginning of the pulse. Since the relaxation of the M at the beginning of the pulse takes place in a shorter time than the duration of the pulse, a flat response is obtained inside the pulse after the first relaxation. This corresponds to a short period during which the $M$ vector is at new equilibrium position, $\theta_{2}$. After $10 \mathrm{~ns}$, at the end of the applied pulse, the only field acting on the sample is the bias field. Hence, $M$ will move back from the perturbed direction $\theta_{2}$ to its initial equilibrium direction, again via a precession around the bias field. This corresponds to the second relaxation signal at the end of the pulse. The experimental evidence of these relaxations is very important because they are the basis of ultra-fast magnetization reversal concepts proposed previously.

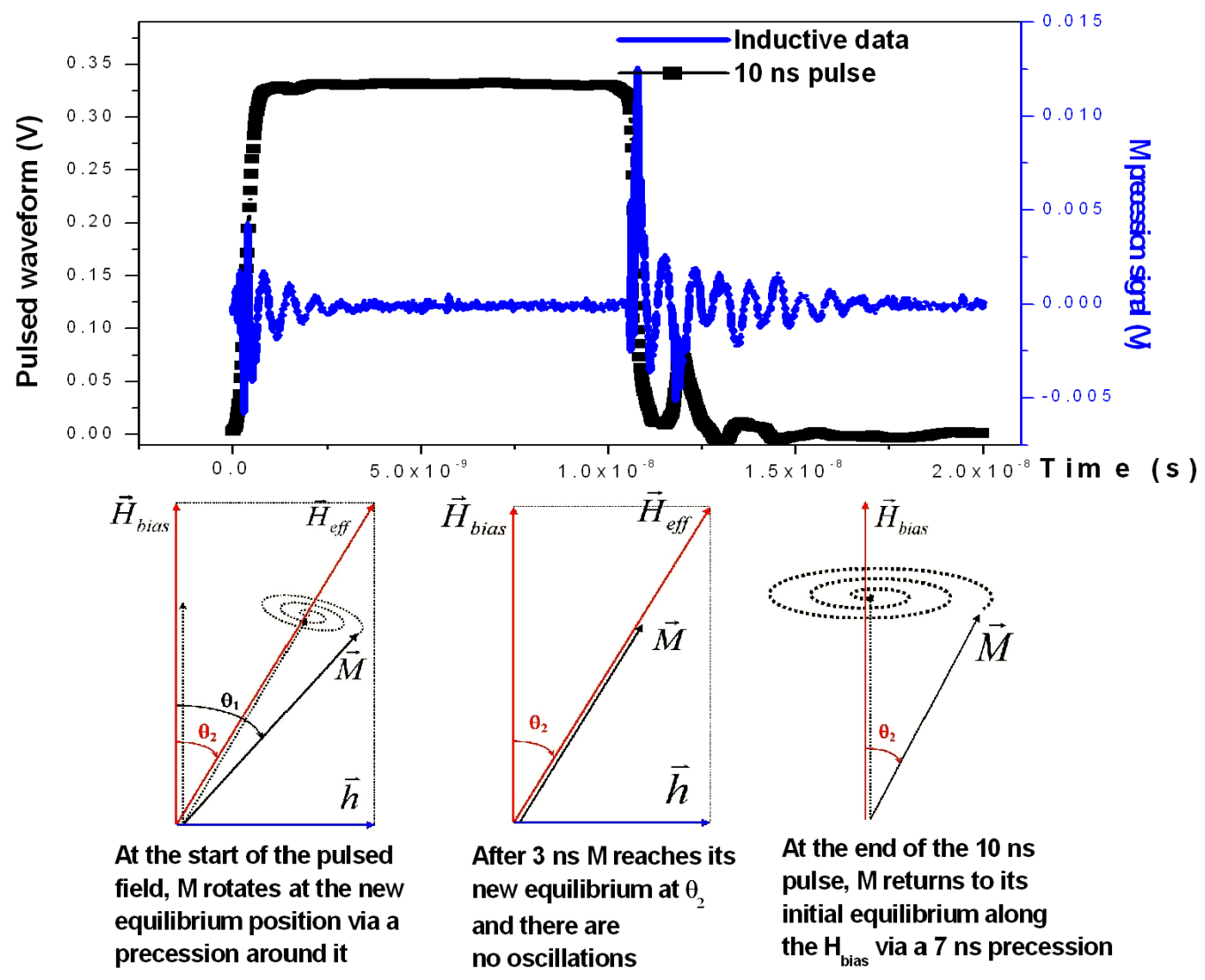

Figure 9. Top figure shows the inductive relaxation response and the $10 \mathrm{~ns}$ pulse. The bottom diagrams show the vectorial diagrams of the $M$ vector as it undergoes the first precession, equilibrium state inside the pulse and the second precession at the end of the pulse, respectively. 
For example, if the duration of the applied pulse field is comparable or shorter than that of the first relaxation, then the oscillation of the first precession can interfere with the oscillation of the second one [14]. This interaction was exploited experimentally $[4,7,14]$ and theoretically $[15,16]$ in defining various ultra-fast magnetization reversal processes.

\section{Conclusions}

We report the design and construction of an improved pulsed inductive microwave magnetometer optimized for fast dynamic magnetization metrologies of soft magnetic materials. The instrument shows unique capabilities in terms of versatility, fast data acquisition, repeatability, reduction of required equipment, simplifications of the measurement procedures and cost reductions. We believe that some of the improvements and benefits of this instrument arise from the fact that the CPW cavity has been designed and fully optimized for this particular experiment, as well as the efficient LabView automation of the experimental parts and measurement procedures. The instrument has been tested on NiFe thin film samples. We show that, depending on the duration of the applied excitation pulse, the inductive relaxation response can show one relaxation at the end of the pulse or two relaxation processes for larger pulse durations. The two relaxations at the beginning and the end of the applied pulse are understood and well-known, although we do not recall any papers showing experimental data of the two precessions acquired in real time, simultaneously.

\section{References}

[1]. L. Landau, E. Lifschitz, Phys. Z. Sowjetunion, 8, 153 (1953)

[2]. T.L. Gilbert, Phys. Rev. 100, 1243 (1955)

[3]. M. Bauer, J. Fassbender, B. Hillebrands, R.L. Stamps, Phys. Rev. B vol. 61, No. 5, 3410-3416 (2000)

[4]. M. Bauer, R. Lopusnik, J. Fassbender, B. Hillebrands, Appl. Phys. Lett. Vol 76, No. 19, 2758 $2760(2000)$

[5]. A.B. Kos, T.J. Silva, P. Kabos, Rev. Sci. Instr. Vol. 73, No. 10, 3563-3569 (2002)

[6]. T.J. Silva, C.S.Lee, T.M. Crawford, C.T. Rogers, J. Appl. Phys. Vol. 85, No. 11, 7849-7862 (1999)

[7]. Th. Gerrits, H.A.M. van den Berg, J. Hohlfeld, L. Bär, Th. Rasing, Nature, 418, 509-511 (2002)

[8]. Th. Gerrits, H.A.M. van den Berg, J. Hohlfeld, O. Gielkens, L. Bär, Th. Rasing, Journal of Magnetism and Magnetic Materials, 240, 283-286 (2002)

[9]. R. Salikhov, R. Abrudan, F. Brussing, St. Buschhorn, M. Ewerlin, D. Mishra, F. Radu, I.A. Garifullin, H. Zabel, Appl. Phys. Letter. 99, 092509 (2011)

[10]. C.H. Back, H.C. Siegmann, Rasing, Journal of Magnetism and Magnetic Materials, 200, 774-785 (1999)

[11]. H.W. Schumacher, C. Chappert, P. Crozat, R.C. Sousa, P.P. Freitas, J. Appl. Phys. 91, 8043-8045 (2002)

[12]. H.W. Schumacher, C. Chappert, R.C. Sousa , P.P. Freitas, J. Miltat, Phys. Rev. Letter. 90, 017204 (2003)

[13]. M. Vopsaroiu, M.J. Thwaites, S. Rand, P.J. Grundy, K. O’Grady, IEEE Trans. Magn. 40, 2443 (2004)

[14]. T.M. Crawford, P. Kabos, T.J. Silva, Appl. Phys. Lett. Vol. 76, No. 15, 2113-2115 (2000)

[15]. M. Bauer, J. Fassbender, B. Hillebrands, R.L. Stamps, Phys. Rev. B. 61, 3410 - 3416, (2000)

[16]. W.K. Hiebert, G.E. Ballentine, M.R. Freeman, Phys., Rev. B. 65, 140404 (2002) 